\title{
Identificação e remoção cirúrgica de dentes supranumerários: relato de caso
}

Identification and surgical removal of supernumerary teeth: case report

Identificación y extracción quirúrgica de dientes supernumerarios: reporte de caso

Ana Beatriz Rodrigues MOURA ${ }^{1}$

Fabiana Larissa Santos de MEDEIROS ${ }^{1}$

Antônio Pereira de ARAÚJO NETO ${ }^{1}$

Lígia Virgínio FERNANDES ${ }^{2}$

Rodrigo Alves RIBEIRO ${ }^{3}$

Luiz Guedes de CARVALHO NETO ${ }^{4}$

Bárbara Vanessa de Brito MONTEIRO ${ }^{3}$

${ }^{1}$ Graduação em Odontologia, Universidade Federal de Campina Grande - UFCG, 58708-110 Patos - PB, Brasil

${ }^{2}$ Cirurgiã-Dentista, 58135-000 Esperança-PB, Brasil

${ }^{3}$ Professor(a) Doutor(a) do Curso de Odontologia, Universidade Federal de Campina Grande - UFCG, 58708-110 Patos - PB, Brasil

${ }^{4}$ Cirurgião Bucomaxilofacial, 58428-735 Campina Grande - PB, Brasil

\section{Resumo}

Os dentes supranumerários são aqueles que se desenvolvem nos maxilares além dos dentes de série normal. Esses dentes apresentam maior frequência na dentição permanente e, em alguns casos, se associam a doenças sistêmicas e síndromes. Este trabalho teve como objetivo relatar um caso clínico, enfatizando a importância do diagnóstico precoce de dentes supranumerários para evitar problemas complexos e de difícil solução referentes ao comprometimento na estética por desarmonia do sorriso e ao estabelecimento da correta oclusão. Paciente leucoderma, do sexo masculino, 9 anos de idade, buscou atendimento acompanhado do responsável, tendo como queixa principal a existência de um "dente atrofiado" entre os incisivos centrais superiores e um comprometimento na estética por desarmonia do sorriso. Realizada a anamnese, procedeuse ao exame clínico, sendo constatada a presença de mesiodente, acarretando diastema entre os incisivos e vestibularização acentuada do elemento 21, contribuindo para uma ausência de selamento labial adequado. Foram realizadas radiografias periapicais da área, uma radiografia oclusal e uma ortopantomográfica. Foi observado que além da presença de um elemento supranumerário na região anterior (mesiodente), havia outro dente supranumerário incluso na região ântero-mediana do palato. Baseando-se nos exames clínico e radiográfico, optou-se pela remoção cirúrgica dos supranumerários. Os dentes supranumerários são uma anomalia de número dentário que podem se apresentar irrompidos ou inclusos e, além disso, podem relacionar-se com síndromes ou doenças sistêmicas. Dessa forma, observa-se a necessidade de um diagnóstico preciso e, na maior parte dos casos, remoção cirúrgica dos dentes excedentes.

Descritores: Dente Supranumerário; Diagnóstico; Cirurgia Bucal.

\section{Abstract}

Supernumerary teeth are those that develop in the jaws in addition to normal teeth. These teeth are more frequent in permanent dentition and, in some cases, are associated with systemic diseases and syndromes. This study aimed to report a clinical case, emphasizing the importance of early diagnosis of supernumerary teeth to avoid complex and difficult-to-solve problems related to impaired aesthetics due to smile disharmony and the establishment of correct occlusion. Leukoderma patient, male, 9 years old, sought care accompanied by the responsible, having as main complaint the existence of a "atrophied tooth" between the upper central incisors and a compromise in aesthetics due to disharmony of the smile. After the anamnesis was performed, a clinical examination was performed, and the presence of a mesiodent was verified, causing diastema between the incisors and marked vestibularization of the element 21 , contributing to an absence of adequate lip sealing. Periapical radiographs of the area, an occlusal radiography and an orthopantomography were performed. It was observed that in addition to the presence of a supernumerary element in the anterior (mesiodent) region, there was another supernumerary tooth included in the anteromedian region of the palate. Based on the clinical and radiographic exams, we opted for the surgical removal of supernumeraries. Supernumerary teeth are a dental number anomaly that may appear erupted or included and, in addition, may be related to syndromes or systemic diseases. Thus, there is a need for an accurate diagnosis and, in most cases, surgical removal of excess teeth.

Descriptors: Tooth, Supernumerary; Diagnosis; Surgery, Oral.

\section{Resumen}

Los dientes supernumerarios son aquellos que se desarrollan en los maxilares además de los dientes de serie normal. Esos dientes presentan mayor frecuencia en la dentición permanente y, en algunos casos, se asocian a las enfermedades sistémicas y síndromes. Este trabajo tuvo como objetivo informar un caso clínico, enfatizando la importancia del diagnóstico precoz de dientes supernumerarios para evitar problemas complejos y de difícil solución referentes al comprometimiento en la estética por desarmonía de la sonrisa y al estabelecimiento de la correcta oclusión. Paciente leucoderma, del género masculino, 9 años, buscó atención médica acompañado de su responsable, teniendo como queja principal la existencia de un "diente atrofiado" entre los incisivos centrales superiores y un comprometimiento en la estética por desarmonía de la sonrisa. Realizada la anamnesis, se realizó el examen clínico, donde fue comprobada la presencia de mesiodente, acarreando diastema entre los incisivos y vestibularización acentuada del elemento 21, contribuyendo para una ausencia de sellamiento labial adecuado. Fueron realizadas radiografías periapicales del área, una radiografía oclusal y una ortopantomográfica. Se ha observado que además de la presencia de un elemento supernumerario en la región anterior (mesiodente), había otro diente supernumerario incluido en la región antero medial del palatino. Basándose en el examen clínico y radiográfico, se decidió por la remoción quirúrgica de los supernumerarios. Los dientes supernumerarios son una anomalía de número dentario que pueden presentarse irrumpidos o incluidos y, además de eso, pueden relacionarse con síndromes o enfermedades sistémicas. Así, se observa la necesidad de un diagnóstico preciso y, en la mayor parte de los casos, remoción quirúrgica de los dientes excedentes.

Descriptores: Diente Supernumerario; Diagnóstico; Cirurgía Bucal.

INTRODUÇÃO

Os dentes supranumerários são aqueles que se desenvolvem nos maxilares além dos dentes de série normal. Esses dentes apresentam maior frequência na dentição permanente e, em alguns casos, associam-se a doenças sistêmicas e síndromes. Geralmente, a 
incidência desses elementos dentários é maior na maxila, com prevalência mais acentuada na região anterior. A presença de dentes supranumerários pode causar alterações funcionais e estéticas, provocando complicações na erupção do dente permanente da área afetada ${ }^{1}$.

O surgimento de dentes supranumerários é o resultado de anomalias congênitas do desenvolvimento do número de dentes $^{2}$. Essa patologia pode causar várias complicações, como: apinhamento dentário, impactação de dentes permanentes, retardo na erupção, deslocamento ou rotação dentária, formação de diastemas, desenvolvimento de lesões císticas, reabsorção radicular de dentes adjacentes, perda de espaço e irrompimento de dentes supranumerários na cavidade nasal ${ }^{3-5}$.

Esses dentes são considerados como dentes de desenvolvimento anormal que podem ocorrer tanto na dentição decídua como na dentição permanente. No entanto, a etiologia dos dentes supranumerários ainda permanece desconhecida. Eles podem ser únicos ou múltiplos, uni ou bilaterais, permanecer impactados ou erupcionar na cavidade bucal. Sabe-se que o sexo masculino é o gênero mais prevalente e frequentemente os dentes supranumerários são diagnosticados em exames de rotina, tendo em vista que, na maioria dos casos, são assintomáticos. Quando diagnosticados, a remoção cirúrgica é indicada a fim de que não provoque interferência na dentição permanente ${ }^{1}$.

O objetivo deste trabalho foi apresentar o relato de um caso clínico no qual uma criança de 9 anos de idade exibiu comprometimento na estética por desarmonia do sorriso e problemas na oclusão devido a presença de dentes supranumerários.

CASO CLÍNICO

Paciente leucoderma, do sexo
masculino, 9 anos de idade, buscou atendimento odontológico, acompanhado do responsável, tendo como queixa principal a existência de um "dente atrofiado" entre os incisivos centrais superiores e um comprometimento na estética por desarmonia do sorriso. Realizada a anamnese, procedeu-se ao exame clínico, sendo constatada a presença de mesiodente, acarretando diastema entre os incisivos e vestibularização acentuada do elemento 21 (Figura 1A), contribuindo para uma ausência de selamento labial adequado. Foram realizadas radiografias periapicais da área, uma radiografia oclusal e uma ortopantomográfica. Foi observado que além da presença de um elemento supranumerário na região anterior (mesiodente), havia um outro dente supranumerário incluso na região ânteromediana do palato. Pela radiografia oclusal, a posição do supranumerário incluso não pôde ser bem estabelecida, porém ao utilizar a técnica de Clark distalizando o ponto de entrada dos raios $X$ verificou-se melhor dissociação das estruturas e foi constatado o aspecto de "baioneta", coroa bífida, bem como aspecto não conclusivo de geminação. Também se observou a relação de proximidade no terço apical do elemento 11 (Figura 1B). Foi constatada a inversão do dente, estando à coroa projetandose para as fossas nasais. Baseando-se nos exames clínico e radiográfico, optou-se pela remoção cirúrgica dos supranumerários. Previamente, foi planejada e executada uma placa de acrílico que traria maior conforto pósoperatório ao paciente (Figura 1C). Para o procedimento cirúrgico, inicialmente foi realizada antissepsia perioral com iodopovidona, em seguida antissepsia intra oral com clorexidina $0,12 \%$, colocação de campo fenestrado e anestesia local com Citanest $\AA$ 3\% (Dentsply Ind. Com. Ltda., Petrópolis, RJ, Brasil). Logo após iniciou-se o procedimento cirúrgico com sindesmotomia peridentária e exodontia do supranumerário utilizando-se alavanca, tomando-se o devido cuidado para não ocorrer luxação dos elementos adjacentes. Realizou-se limpeza da cavidade cirúrgica com soro fisiológico a $0,9 \%$, e finalmente foi realizada sutura com fio de seda no 3,0 e prescrição de dipirona 500mg via oral de 6 em 6 horas, em caso de sintomatologia dolorosa nas primeiras horas, além de bochecho após 30 minutos da escovação com clorexidina $0,12 \%$. O período pós-operatório decorreu dentro dos padrões de normalidade, nenhuma alteração na dentição foi observada e os pontos de sutura foram retirados após uma semana. Posteriormente, em uma sessão seguinte, realizaram-se os mesmos procedimentos assépticos descritos na primeira etapa $e$ anestesia local na região de forame incisivo e nervos palatinos maiores e menores para bloqueio sensorial da região palatina. Foi realizada incisão interpapilar com bisturi de lâmina no 15,0, deslocamento mucoperiostal com sindesmótomo e remoção da tábua óssea com auxílio de broca cirúrgica 702 até a visualização do supramumerário incluso (Figura 1D). O dente foi luxado e removido com auxílio da alavanca. Após a remoção, fez-se a curetagem da loja cirúrgica, irrigação com soro fisiológico, reposicionamento do retalho cirúrgico, sutura interpapilar com fio de seda no 3,0 e colocação de placa de acrílico para 
proteção da ferida cirúrgica. Como terapia medicamentosa foi prescrito azitromicina $500 \mathrm{mg}$ via oral uma vez ao dia, dipirona $500 \mathrm{mg}$ via oral de 6 em 6 horas e bochecho após 30 minutos da escovação com clorexidina $0,12 \%$. A sutura e a placa de acrílico foram removidas após uma semana, sendo observado cicatrização satisfatória e movimentação palatina do dente 21, diminuindo sua anormal vestibularição inicial (Figura 1E). Por fim, o paciente foi encaminhado para avaliação ortodôntica.

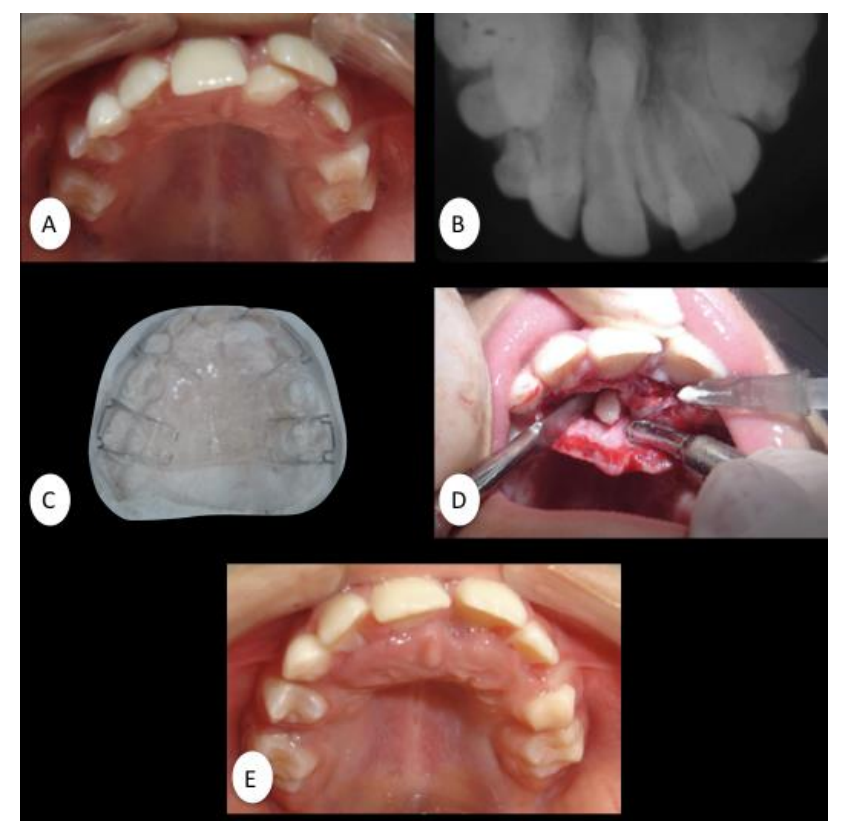

Figura 1: A. Aspecto clínico inicial. B. Radiografia oclusal que permitiu a identificação do supranumerário incluso no palato duro. C. Placa de acrílico confeccionada previamente aos procedimentos cirúrgicos (D), visando maior conforto pósoperatório do paciente. E. Aspecto final, após a cicatrização.

DISCUSSÃO

Os dentes supranumerários podem ocorrer em qualquer região da cavidade oral ${ }^{6}$, podendo ser localizados tanto na maxila como na mandíbula, impactados ou erupcionados, uni ou bilateralmente e podendo ainda ser apenas um ou vários dentes ${ }^{7}$. No presente relato, foram identificados dois dentes supranumerários, sendo um deles um mesiodente. Segundo a literatura, os mesiodentes podem estar presentes devido à síndromes como a de Gardner, ou em pacientes com malformações orofaciais como fenda palatina e labial $^{8}$; mas também podem se apresentarem de forma isolada, conforme o caso clínico aqui relatado.

A presença de dentes supranumerários na porção anterior de maxila apresenta várias complicações funcionais e estéticas, dentre elas podemos citar a rotação, deslocamento, alteração no padrão de mastigação e oclusão, além de diastemas entre os incisivos; dentre outras $^{9}$. Neste caso, o paciente procurou atendimento devido ao comprometimento estético, por ter um sorriso desarmonioso, apresentando além de diastema entre os incisivos, a vestibularização do elemento 21.

Em um estudo com 55 crianças abaixo de 14 anos que apresentavam mesiodentes, encontrou-se uma predominância de pacientes do gênero masculino, sendo estes duas vezes mais afetados do que pacientes do gênero feminino. Em toda a amostra, após avaliação clínica e radiográfica, foi observada a necessidade de intervenção cirúrgica. Em $45,45 \%$ da amostra, foi constatada a necessidade de correção ortodôntica após a cirurgia $^{10}$. Os achados deste estudo são condizentes com o que foi observado neste caso clínico, pois apresentamos aqui um paciente do gênero masculino, com dentes supranumerários que necessitou de intervenção cirúrgica e posterior avaliação ortodôntica.

Tendo em vista todas as complicações diante de um dente supranumerário, o diagnóstico precoce permite ao profissional realizar um tratamento mais conservador ${ }^{11}$. No caso aqui relatado, foi verificada a importância do exame radiográfico para a detecção do dente supranumerário que se encontrava incluso no palato. Dessa forma, é importante que na fase da dentição mista, os profissionais adotem condutas rotineiras e solicitem exames complementares que auxiliem em um diagnóstico mais preciso, permitindo um melhor prognóstico e possibilitando ao paciente a diminuição de ocorrências e de complicações futuras ${ }^{1}$.

A escolha para o tratamento de dentes supranumerários é a remoção cirúrgica, porém a recomendação do tratamento cirúrgico ou para acompanhamento clínico-radiográfico irá depender da idade do paciente e da possibilidade de danos aos tecidos adjacentes e elementos dentários ${ }^{12-15}$. No presente caso, a remoção cirúrgica foi adotada, principalmente devido ao deslocamento dos dentes permanentes em decorrência do mesiodente, acarretando prejuízo estético.

O outro dente supranumerário incluso no palato, também teve indicação de remoção cirúrgica. Sabe-se que devido às características anatômicas e histológicas do palato duro, as intervenções cirúrgicas tendem a apresentar pós-operatório incômodo. Assim, optou-se pela utilização da placa de acrílico imediatamente após a cirurgia, como forma de se prover uma barreira física e isolamento da região suturada, propiciando assim um maior conforto pósoperatório para o paciente.

De forma geral, a conduta de remover os dentes surpranumerários foi adotada com o 
intuito de harmonizar o sorriso, devolver estética e gerar menos transtornos à criança. $O$ caso nos mostra que além da anamnese, exame clínico e diagnóstico; os exames complementares, como a radiografia e o plano de tratamento são imprescindíveis para 0 sucesso clínico. Para que o paciente seja beneficiado, é importante o conhecimento por parte dos profissionais das características desta anomalia, bem como do tratamento adequado, além do ponto de vista estético e o impacto social que esta anomalia pode causar em seu estilo de vida, é importante que este tratamento seja o mais conservador possível, possibilitando assim um pós-operatório satisfatório para 0 paciente, assim como para o cirurgião-dentista.

\section{REFERÊNCIAS}

1. Nunes KM, Medeiros MV, Ceretta LB, Simões PW, Azambuja FG, Sônego FGF et al. Dente supranumerário: revisão bibliográfica e relato de caso clínico. Rev Odontol Univ Sao Paulo. 2015;27:72-81.

2. Mukhopadhyay S. Mesiodens: a clinical and radiographic study in children. J Indian Soc Pedod Prev Dent. 2011;29:34-8

3. Cunha Filho JJ, Puricelli E, Hennigen TW, Leite MGT, Pereira MA, Martins GL. Ocorrência de dentes supranumeráriosem pacientes do serviço de Cirurgia e Traumatologia BucoMaxilo--Facial, Faculdade de Odontologia da UFRGS, no período de 1998 a 2001.Rev Fac Odontol Porto Alegre. 2002;43:27-34.

4. Fernandes AV, Rocha NS, Almeida RAC, Silva EDO, Vasconcelos BCE. Quarto molar incluso: relato de caso. Rev cir traumatol buco-maxilofac. 2005;5:61-6.

5. Leite Segundo AV, Faria DLB, Silva UH, Vieira ÍTA. Estudo epidemiológico de dentes supranumerários diagnosticados pela radiografia panorâmica. Rev cir traumatol bucomaxilo-fac. 2006;6:53-6.

6. Parolia A, Kundabala M, Dahal M, Mohan M, Thomas MS. Management of supernumerary teeth. J Conserv Dent. 2011;14:221-24.

7. Schmuckli R, Lipowsky C, Peltomäki T. Prevalence and morphology of supernumerary teeth in the population of a swiss community. Schweiz Monatsschr Zahnmed. 2012;120: 987-90.

8. Marchetti G, Oliveira RV. Mesiodens - dentes supranumerários: diagnóstico, causas e tratamento. Uningá Review. 2015;24:19-23.

9. Wang XP, Fan J. Molecular genetics of supernumerary tooth formation. Genesis. 2011; 49:261-77.

10. Ramesh K, Venkataraghavan K, Kunjappan S, Ramesh M. Mesiodens: a clinical and radiographic study of 82 teeth in 55 children below 14 years. J Pharm BioAllied Sci. 2013;5:60-2.

11. Stringhini Junior E, Stang B, Oliveira LB. Dentes supranumerários impactados: relato de caso clínico. Rev Assoc Paul Cir Dent. 2015;69:89-94.

12. Garvey MT, BarryHJ,Blake M. Supernumerary teeth-an over view of classification, diagnosis and management. J Can Dent Assoc. 1999;65:612-16.

13. Mason C, Rule D, Hopper C. Multiple supernumeraries: the importance of clinical and radiographic follow-up. Dentomaxillofac Radiol. 1996;25:109-13.

14. Almeida CM, Pereira EL, Carvalho DA, Silva Júnior SE, Lima VPR, Araújo Filho JCWP. Exodontia de elemento supranumerário incluso e impactado localizado na mandíbula. Arch Health Invest. 2018:7 (Special Issue 1):27.

15. Teslenco VB, Gaetti Jardim EC, Silva JCL. Supranumerários bilaterais em mandíbula: relato de caso. Arch Health Invest. 2017:6 (3):110-14.

\section{CONFLITO DE INTERESSES}

Os autores declaram não haver conflitos de interesse

\section{AUTOR PARA CORRESPONDÊNCIA}

\section{Bárbara Vanessa de Brito Monteiro}

Universidade Federal de Campina Grande

Centro de Saúde e Tecnologia Rural

Unidade Acadêmica de Ciências Biologicas

Av. Universitária, s/n. Bairro Santa Cecília.

58708-110 Patos - PB, Brasil.

E-mail: barbaravbm@hotmail.com

Submetido em 05/04/2020

Aceito em 23/10/2020 